\title{
DYNAMICS OF RETICULATED STRUCTURES: EVIDENCE OF ATYPICAL GYRATION MODES
}

\section{Chesnais, S. Hans, \& C. Boutin*}

École Nationale des Travaux Publics de l'État, Université de Lyon, DGCB, FRE CNRS 3237, Lyon, France

*Address all correspondence to C. Boutin, E-mail: claude.boutin@entpe.fr

This paper deals with the dynamic behavior of periodic reticulated beams made of symmetric unbraced framed cells. Such archetypical cells can present a high contrast between shear and compression deformabilities that opens the possibility of enriched local kinematics. Through the homogenization method of periodic discrete media associated with a systematic use of scaling, the existence of atypical gyration modes is established theoretically. These latter modes appear when the elastic moment is balanced by the rotation inertia, conversely to "natural" modes where the elastic force is balanced by the translation inertia. A generalized beam modeling including both "natural" and gyration modes is proposed and discussed through a dimensional analysis. The results are confirmed on numerical examples.

KEY WORDS: reticulated structures, homogenization, structural dynamics, micromorphic media, gyration modes, generalized beam

\section{INTRODUCTION}

This paper deals with the macroscopic dynamic behavior of periodic reticulated structures widely encountered in mechanical engineering. Periodic lattices have been studied through various approaches such as transfer matrix, variational approach (Kerr and Accorsi, 1985), and finite-difference operator (Noor, 1988). Asymptotic methods of homogenization (Sanchez-Palencia, 1980) initially developed for periodic media, were extended to multiple parameters and scale changes by Cioranescu and Saint Jean Paulin (2010) and adapted to periodic discrete structures by Caillerie et al. (1989), and then by Moreau and Caillerie (1998). Unbraced framed structures have been studied in structural dynamics, for instance by Skattum (1971). All those studies aim to relate the features of the basic cell and the global behavior.

The morphology of reticulated beams is such that the basic cells can present a high contrast between shear and compression deformabilities (conversely to "massive" beams). This opens the possibility of enriched local kinematics involving phenomena of global rotation, inner deformation or inner resonance, according to studied configuration and frequency range (Hans and Boutin, 2008). A numerical illustration of these atypical situations is given in Fig. 1, which shows some unusual macroscopic modes (Chesnais, 2010).

This paper addresses the theoretical evidence of atypical gyration modes. These latter modes appear when the elastic moment is balanced by the rotation inertia, conversely to "natural" modes where the elastic force is balanced by the translation inertia. We proceed in two steps. First, analysis is performed on the archetypical case of symmetric unbraced framed cells (Boutin and Hans, 2003; Hans and Boutin, 2008). Assuming the cell size is small compared to the wavelength, the homogenization method of periodic discrete media leads to the macro-behavior at the leading order. Second, the results are extended to a wider class of reticulated frames. A generalized beam modeling including both natural and gyration modes is established via a dimensional analysis approach.

To our knowledge very few works are devoted to such topics. The possibility of gyration modes has been mentioned by Skattum (1971) and partially treated in some study cases. However, a comprehensive analytical approach 

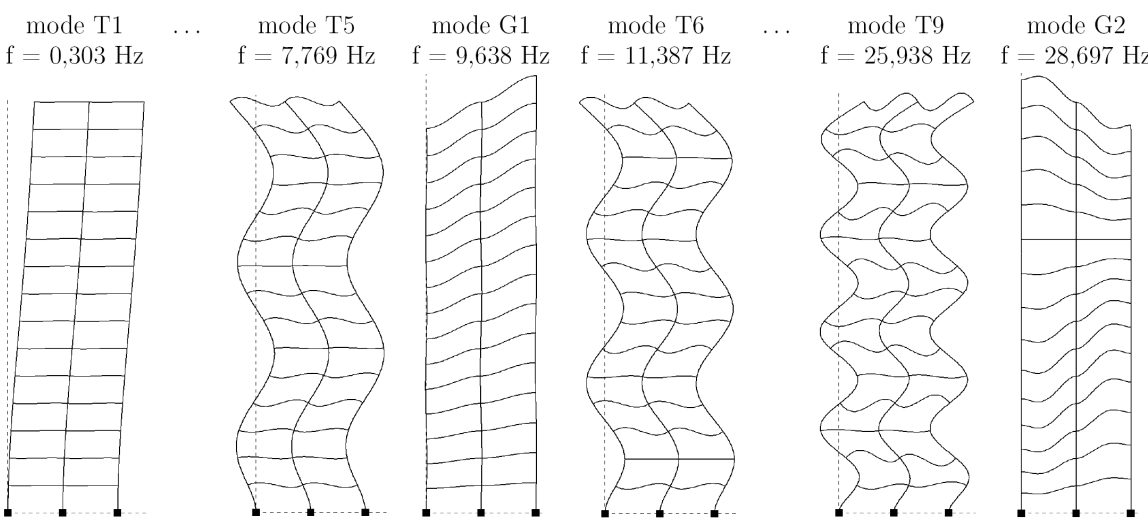

$\mathrm{mo}$
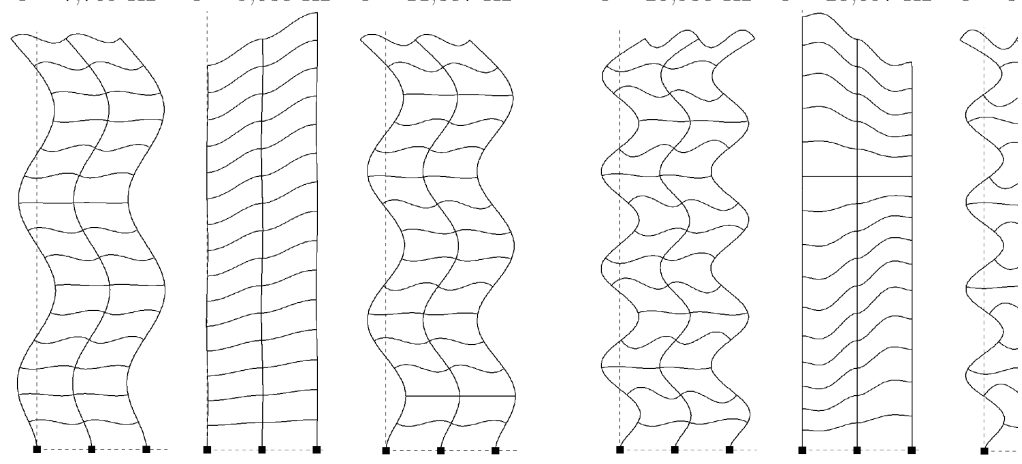

FIG. 1: Examples of typical (T) and atypical (G) mode shapes of framed structures.

aiming at understanding and modeling gyration modes is not yet available in a general framework. The present paper provides a contribution in this field.

The paper is organized as follows. Section 2 gives an overview of the method. In Section 3 the different types of natural transverse modes (even if the beam behavior is not classical) are presented and discussed in a particular case. Section 4 is devoted to gyration vibrations, governed by the balance of elastic moment and rotation inertia. In Section 5, a generalized modeling is proposed, discussed through a dimensional analysis and illustrated numerically.

\section{OVERVIEW OF DISCRETE HOMOGENIZATION AND STUDIED STRUCTURES}

An outline of the method is given hereafter, for a detailed presentation one may refer to Hans and Boutin (2008). The analysis of periodic beam lattices is performed in two steps (Tollenaere and Caillerie, 1998).

\subsection{Discretization}

The studied structures (Fig. 2) are made of plates behaving as Euler-Bernoulli beams in out-of-plane motion, and assembled with rigid connections. The motions of each extremity connected to the same node are identical and define
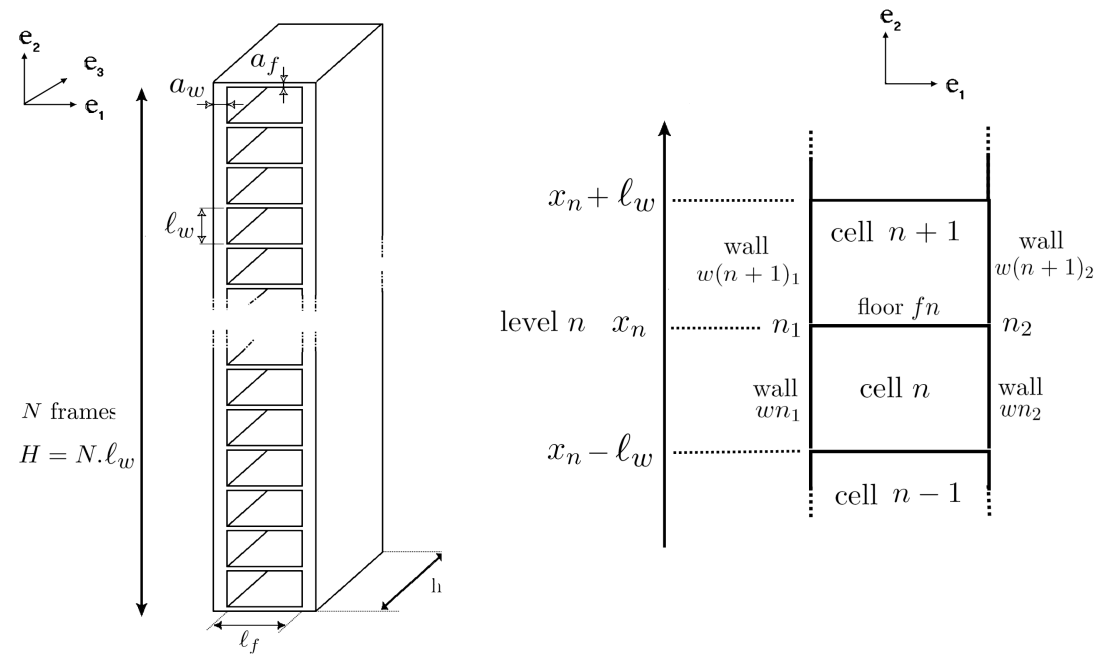

FIG. 2: The class of unbraced framed structures (left) and the basic frame and notations (right). 
the discrete nodal kinematic variables of the system. The discretization consists in integrating the dynamic balance (in a harmonic regime) of the beams, the unknown displacements and rotations at their extremities taken as boundary conditions. Forces applied by an element on its extremities are then expressed as functions of the nodal variables. The balance of each element being satisfied, the balance of the whole structure is rigorously reduced to the balance of the nodes.

\subsection{Homogenization}

The key assumption is that cell size $\ell$ is small compared to the characteristic size $L$ of the vibrations of the structure. Thus, $\epsilon=\ell / L \ll 1$. The existence of a macro-scale is expressed by means of a macro-space variable $x$. The physical variables are continuous functions of $x$ coinciding with the discrete variables at any node; e.g., $U_{\epsilon}(x=$ $\left.x_{n}\right)=U($ node $n)$. These quantities, assumed to converge when $\epsilon$ tends to zero, are expanded in powers of $\epsilon$ : $U_{\epsilon}(x)=U^{0}(x)+\epsilon U^{1}(x)+\epsilon^{2} U^{2}(x)+\ldots$ Similarly, all unknowns, including the modal frequency, are expanded in powers of $\epsilon$. As $\ell=\epsilon L$ is small with respect to $x$, the variations of the variables between neighboring nodes are expressed using Taylor's series, which introduces the macroscopic derivatives. To account for the local physics, the geometrical and mechanical characteristics of the elements are scaled according to the powers of $\epsilon$. As for modal frequencies, the scaling is imposed by the balance of elastic and inertia forces at the macro-level. The scaling insures that each effect appears at the same order whatever the $\epsilon$ value is. Therefore, the same physics is kept when $\epsilon \rightarrow 0$; i.e., for the homogenized model. Finally, the expansions are introduced in the nodal balances. Those relations, valid for any small $\epsilon$, lead for each $\epsilon$-order to balance equations whose resolution defines the macroscopic equations.

\subsection{Inner Quasi-Statics and Inner Dynamics}

In general, the scale separation requires wavelengths of the compression and bending vibrations generated in each local element to be much longer than the element length at the modal frequency of the global system. Hence, the nodal forces can be developed in Taylor's series with respect to $\epsilon$. This situation corresponds to a quasi-static state at the local scale. The developments presented hereafter are established in this context.

Note that situations where only the compression wavelength is much longer than the length of the elements, while local resonance in bending appears, are also possible. The homogenization still applies through the expansions of the compression forces only and leads to atypical descriptions with inner dynamics. For such situations, out of the focus of this paper, one may refer to Boutin et al. (2009), Chesnais et al. (2007), Chesnais (2010).

\subsection{Natural and Unusual Transverse Vibrations}

According to the properties of the cell of the reticulated structures, the homogenization enables to evidence two categories of transverse vibrations related to the nature of the governing dynamic balance.

For natural modes (Section 3), the horizontal elastic force balances the horizontal translation inertia. When the elastic moment is balanced by the rotation inertia one obtains the unusual gyration modes (Section 4).

\subsection{Structures}

The structures, of height $H=N . \ell$, span $h$, consist in a large number $N$ of identical unbraced frames called cells (Fig. 2). The parameters of horizontal $(i=f)$ and vertical elements $(i=w)$ are: length $\ell_{i}\left(\ell=\ell_{w}\right)$; thickness $a_{i}$; section area $A_{i}=a_{i} h$; inertia $I_{i}=a_{i}^{3} h / 12$ in direction $e_{3}$; density $\rho_{i}$; and elastic modulus $E_{i}$.

At any level $n$, the displacements in the two directions and the rotation $\left(u_{1}, u_{2}, \theta\right)$ of the two nodes can be replaced by (1) three variables associated with the rigid body motion of level $n$ : the mean transverse displacements, $U(n)$ along $e_{1}, V(n)$ along $e_{2}$, and the rotation $\alpha(n)$ (differential vertical nodal motion divided by $\ell_{f}$ ) and (2) three variables corresponding to the deformation of level $n$ : the mean and differential rotations of the nodes, $\theta(n)$ and $\Phi(n)$, and the transverse dilatation $\Delta(n)$ (cf. Fig. 3). Because of the longitudinal symmetry, the transverse kinematics associated with $(U, \alpha, \theta)$ is uncoupled with the longitudinal kinematics (driven by $V, \Phi, \Delta$; not studied here). 


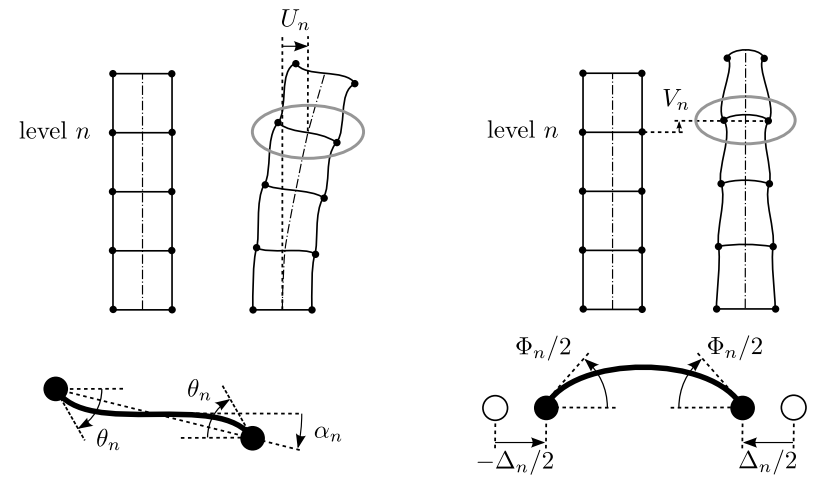

FIG. 3: Decoupling of transverse (left) and longitudinal (right) kinematics.

\section{NATURAL TRANSVERSE VIBRATIONS}

For natural transverse vibrations, the horizontal elastic force balances the horizontal translation inertia and the frequency range is such that the cell behaves quasi-statically (lower frequencies would lead to a static regime). The possible beam-like behaviors were established by varying the properties of the basic frame elements by Hans and Boutin (2008) to which one may refer for a precise analysis. The synthesis of the different macro-behaviors shows that only three mechanisms-shear, global bending, and inner bending-govern the physics at the macro-scale. Each of them is associated with an elastic cell parameter of stiffness: in shear $K$, in global bending $E_{w} I$, and in inner bending $E_{w} \mathcal{I}$ ( $\mathcal{I}$ is the effective inner bending inertia). Owing to the quasi-static local state, these parameters are deduced from the elastic properties of elements in statics. For structures as in Fig. 2, they read ( $\Lambda$ stands for the linear mass):

$$
\begin{gathered}
K^{-1}=K_{w}^{-1}+K_{f}^{-1}, \quad K_{w}=24 \frac{E_{w} I_{w}}{\ell_{w}^{2}}, \quad K_{f}=12 \frac{E_{f} I_{f}}{\ell_{w} \ell_{f}}, \quad I=\frac{A_{w} \ell_{f}^{2}}{2}, \quad \mathcal{I}=2 I_{w} \\
\Lambda=\Lambda_{w}+\Lambda_{f}, \quad \Lambda_{w}=\rho_{w} A_{w}, \quad \Lambda_{f}=\rho_{f} A_{f} \frac{\ell_{f}}{\ell_{w}}
\end{gathered}
$$

\subsection{Generic Beam Model}

The generic beam model is governed by

- The beam behavior laws (3) relating macroscopic shear force $T$, global bending moment $M$, and the inner bending moment $\mathcal{M}$ to the kinematic variables $U$ and $\alpha$ (rigid body motion of the section):

$$
T=-K\left(U^{\prime}-\alpha\right), \quad M=-E_{w} I \alpha^{\prime}, \quad \mathcal{M}=-E_{w} \mathcal{I} U^{\prime \prime}
$$

- The force and moment of momentum balance equations:

$$
\left\{\begin{array}{l}
\left(T-\mathcal{M}^{\prime}\right)^{\prime}=\Lambda \omega^{2} U \\
M^{\prime}+T=0
\end{array}\right.
$$

This generalized beam description includes shear and inner bending. The dominating effect(s) that actually drive(s) the effective behavior of a given structure, can be identified through a dimensional analysis. In this aim, we introduce the actual first mode vibration's size $\tilde{L}=2 H / \pi$ (for the $n^{\text {th }}$ mode of a clamped-free beam, $\tilde{L}_{n}=2 H /[(2 n-1) \pi]$ ) and the variables are rewritten as $U=U^{r} U^{*}$ and $\alpha=\alpha^{r} \alpha^{*}$ where the exponent $r$ denotes reference values, and $*$ 


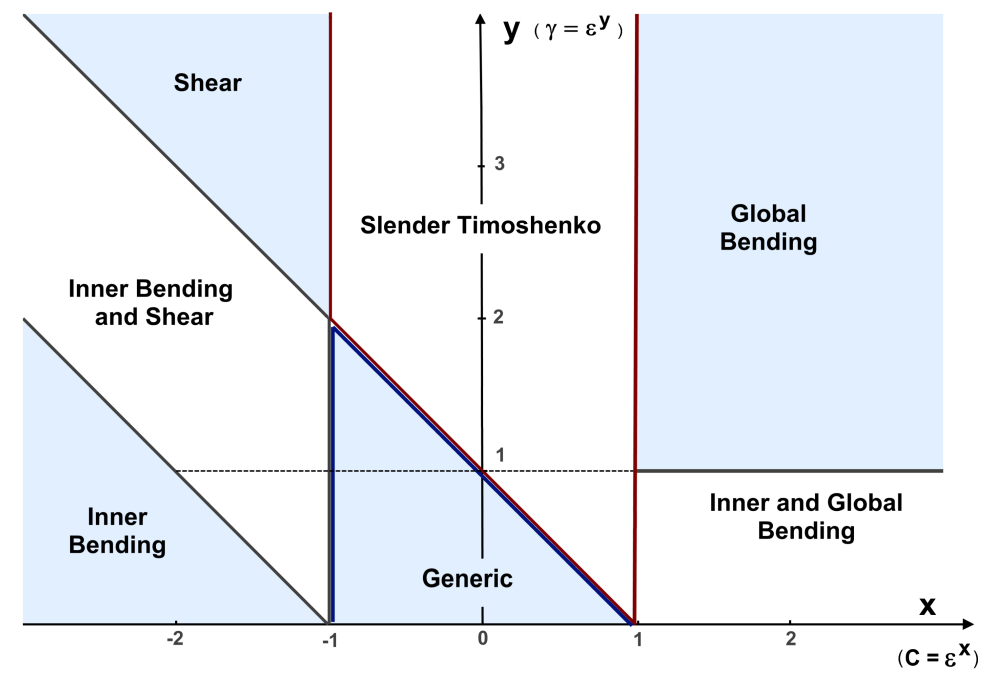

FIG. 4: Map of different kinds of transverse natural behaviors in function of the parameters $C=\tilde{\epsilon}^{x}$ and $\gamma=\tilde{\epsilon}^{y}$, Hans and Boutin (2008). As discussed in Section 5.2, the zoning also applies for the gyration modes: (1) In the domain of Global Bending without shear $C \leq O(\epsilon)$, gyration modes are impossible. (2) In the Generic and Slender Timoshenko domains, $O\left(\epsilon^{-1}\right) \geq C \geq O(\epsilon)$, gyration modes occur provided that $\zeta \leq O(\epsilon)$, and their frequency range is much higher than for natural modes. (3) In the Shear and Inner Bending domains $C \geq O\left(\epsilon^{-1}\right)$ both natural and gyration modes exist and are uncoupled while their frequency range may be common or disjointed.

denotes the dimensionless terms, $O(1)$ by construction. Introducing the expression of the beam efforts (3) and making the change of variables $\boldsymbol{x}=x / \tilde{L}$, set (4) becomes

$$
\left\{\begin{array}{l}
\Omega^{2} U^{*}+U^{*(2)}-C \gamma U^{*(4)}=\left(\tilde{L} \alpha^{r} / U^{r}\right) \alpha^{* \prime} \\
-\alpha^{*}+C \alpha^{*(2)}=-\left(U^{r} / \tilde{L} \alpha^{r}\right) U^{* \prime}
\end{array}\right.
$$

where the numbers in brackets stand for the order of derivative. The dimensionless numbers $C, \gamma(\leq 1$ for common cell geometry) and $\Omega^{2}$ compare, respectively, global bending and shear, inner and global bendings, translation inertia, and shear. They read

$$
C=\frac{E_{w} I}{K \tilde{L}^{2}} ; \quad \gamma=\frac{\mathcal{I}}{I} ; \quad \Omega^{2}=\frac{\Lambda \omega^{2} \tilde{L}^{2}}{K}
$$

Eliminating $\alpha^{*}$ (or $U^{*}$ ) in Eq. (5) gives the differential equation governing $U^{*}$ (or $\left.\alpha^{*}\right)$ :

$$
C \gamma U^{*(6)}-(1+\gamma) U^{*(4)}-\Omega^{2} U^{*(2)}+\frac{\Omega^{2}}{C} U^{*}=O(\tilde{\epsilon})
$$

The term $O(\tilde{\epsilon})$ highlights the fact that Eq. (7) is a zero-order balance and, hence, is only valid up to the accuracy $\tilde{\epsilon}$. Consequently, according to the values of $C, C \gamma$ and $\gamma$ compared to $\tilde{\epsilon}$ powers $(\tilde{\epsilon}=\ell / \tilde{L}=\pi / 2 N)$, Eq. (7) degenerates into simplified forms. The mapping (Fig. 4) gives the validity domain of the seven possible behaviors according to the two parameters $x$ and $y$ defined by $C=\tilde{\epsilon}^{x}$ and $\gamma=\tilde{\epsilon}^{y}$. Note that as the validity of the model requires the scale separation; i.e., $\ell / \tilde{L}_{n}<1$, the maximum number of homogenizable modes of a structure of $N$ cells is at the most $n_{\max }=N / 3$. 


\subsection{An Example: The Slender Timoshenko Beam}

Consider structures for which $C=O(1)$ and $\gamma \leq O(\tilde{\epsilon})$. Then, the terms related to $C \gamma$ and $\gamma$ are negligible in Eq. (7) and the model is a slender Timoshenko beam driven by

$$
U^{*(4)}+\Omega^{2} U^{*(2)}-\frac{\Omega^{2}}{C} U^{*}=O(\tilde{\epsilon})
$$

To show how to reach Eq. (8) by homogenization, consider a structure as shown in Fig. 2 with the floors thicker than the walls:

$$
\frac{a_{w}}{l_{w}}=O(\epsilon) ; \quad \frac{a_{f}}{l_{w}}=O(\sqrt{\epsilon}) ; \quad \frac{\ell_{w}}{\ell_{f}}=O(1) ; \quad \frac{E_{w}}{E_{f}}=O(1)
$$

so that, as required:

$$
C=\frac{E_{w} I}{K_{w} \tilde{L}^{2}}=O\left(\frac{\ell_{w}^{2}}{a_{w}^{2}} \frac{\ell_{w}^{2}}{\tilde{L}^{2}}\right)=O(1) ; \quad \gamma=\frac{2 I_{w}}{I}=O\left(\frac{a_{w}^{2}}{3 \ell_{f}^{2}}\right)=O\left(\epsilon^{2}\right)
$$

The dynamic regime is reached when $\Omega^{2} / C=O(1)$; i.e., since $C=O(1), \Lambda=O\left(\Lambda_{f}\right), K=O\left(K_{w}\right)$, for circular frequency $\omega_{0}=O\left(\tilde{L}^{-1} \sqrt{K_{w} / \Lambda_{f}}\right)=O\left(K_{w} / \sqrt{E_{w} I \Lambda_{f}}\right)$. In that case, the leading order equations obtained by homogenization are

$$
\left\{\begin{array}{l}
-K_{w}\left(U^{0 \prime \prime}-\theta^{0 \prime}\right)=\Lambda_{f} \omega_{0}^{2} U^{0} \\
K_{f}\left(\alpha^{0}-\theta^{0}\right)=0 \\
-E_{w} I \alpha^{0 \prime \prime}-K_{w}\left(U^{0 \prime}-\theta^{0}\right)=0
\end{array}\right.
$$

Equation (10a) expresses the balance of horizontal forces; Eqs. (10b) and (10c) are derived from the balance of both local and global moments at the first two significant orders. Equation (10b) also describes the inner equilibrium of the cell and imposes $\theta^{0}=\alpha^{0}$. Thus, the behavior is described by a differential set that governs the mean transverse motion $U^{0}$ and the section rotation $\alpha^{0}$ :

$$
\left\{\begin{array}{l}
-K_{w}\left(U^{0 \prime \prime}-\alpha^{0 \prime}\right)=\Lambda_{f} \omega_{0}^{2} U^{0} \\
-E_{w} I \alpha^{0 \prime \prime}-K_{w}\left(U^{0 \prime}-\alpha^{0}\right)=0
\end{array}\right.
$$

Eliminating $\alpha^{0}$ provides:

$$
E_{w} I U^{0(4)}+\frac{E_{w} I}{K_{w}} \Lambda_{f} \omega^{2} U^{0(2)}-\Lambda_{f} \omega_{0}^{2} U^{0}=0
$$

which corresponds to Eq. (8); i.e., a degenerated form of Eq. (7) with $\gamma \leq O(\tilde{\epsilon})$. The similarity with Timoshenko beams is obvious when rewriting Eq. (11) with the macro-shear force $\tilde{T}^{0}$ and the global bending moment $\tilde{M}^{0}$ defined in Eq. (3) (here with 0 exponent):

$$
\left\{\begin{array}{l}
T^{0 \prime}=\Lambda_{f} \omega_{0}^{2} U^{0} \\
M^{0 \prime}+T^{0}=0
\end{array}\right.
$$

Two features distinguish Eq. (12) from the Timoshenko description of "massive" beams. First, the shear effect [that comes from the bending of the walls in parallel, Eq. (1)] remains at the leading order even if the reticulated structure is slender. Second, while the translation inertia is significant in the force balance [Eq. (12a)], the rotation inertia is negligible in the moment of momentum balance [Eq. (12b)] (where the effective bending results from the opposite extension-compression of the two walls distant from the floor length). In other words, the translation is in the dynamic regime but the rotation remains in quasi-statics for the studied frequency range. This leads to considering higher frequencies to obtain rotational dynamics. 


\section{ATYPICAL TRANSVERSE VIBRATIONS: GYRATION MODES}

This section is devoted to gyration modes; i.e., transverse modes resulting from the dynamic equilibrium between elastic moment and rotation inertia. As previously mentioned, this requires considering higher frequencies than for natural modes. Consequently, in addition to the change of kinematics, phenomena of inner dynamics could also occur. Here, only situations with local quasi-statics will be investigated. A particular case is first treated, whose results are slightly generalized. Then, the theoretical features of the gyration modes are studied and confirmed through a numerical example.

\subsection{Evidence of Gyration Modes}

To evidence the existence of gyration modes, we come back to the framed structures, whose geometry and parameters are scaled as previously shown in Eq. (9). The frequency range is increased of one order in $\epsilon$; i.e., $\omega_{0}=$ $O\left(\ell^{-1} \sqrt{K_{w} / \Lambda_{f}}\right)$, which remains sufficiently low to insure the quasi-static regime of the cell. As above, the three equations derived by homogenization express (1) the balance of horizontal forces at the leading order [Eq. (13a)], (2) the inner equilibrium of the cell derived from the balance of both local and global moments at the leading order [Eq. (13b)], and (3) the sum of the local and global moments at the next significant order [Eq. (13c)]:

$$
\left\{\begin{array}{l}
0=\Lambda_{f} \omega_{0}^{2} U^{0} \\
K_{f}\left(\alpha^{0}-\theta^{0}\right)=0 \\
-E_{w} I \alpha^{0 \prime \prime}-K_{w}\left(U^{0 \prime}-\theta^{0}\right)=\frac{\rho_{f} A_{f} \ell_{f}^{3}}{420 \ell_{w}} \\
\times \omega_{0}^{2}\left(42 \alpha^{0}-7 \theta^{0}\right)
\end{array}\right.
$$

The comparison of Eqs. (10) with Eqs. (13) shows that the higher frequency leaves the inner equilibrium condition of the cell unchanged (thus, here also the mean rotation of the nodes matches the section rotation; i.e., $\theta^{0}=\alpha^{0}$ ). Conversely, the increased order of magnitude of inertia terms implies that $\Lambda_{f} \omega_{0}^{2} U^{0}$ cannot be balanced by horizontal elastic forces; thus, the section translation vanishes, $U^{0}=0$. In parallel, the rotation inertia now appears in the moment of momentum balance. After eliminating $\theta^{0}$, the macroscopic behavior at the leading order is described by the following differential equation of the second degree:

$$
\left\{\begin{array}{l}
-E_{w} I \alpha^{0 \prime \prime}+K_{w} \alpha^{0}=J_{f} \omega_{0}^{2} \alpha^{0} \\
\theta^{0}=\alpha^{0} ; \quad U^{0}=0 ; \quad J_{f}=\frac{\rho_{f} A_{f} \ell_{f}^{3}}{12 \ell_{w}}
\end{array}\right.
$$

This is an atypical gyration beam model fully driven by the section rotation $\alpha^{0}$ without lateral translation (more precisely, one shows that the first nonvanishing translation is of the second-order $\left.\epsilon^{2} U^{2}=\left[K_{w} / \Lambda_{f} \omega_{0}^{2}\right] \alpha^{0 \prime}\right)$. The gyration dynamics is governed by the mechanism of opposite traction-compression of vertical elements (the elastic parameter is the global bending stiffness $E_{w} I$ ), the shear of the cell (stiffness $K_{w}$ ) acting as an inner elastic source of moment, and the classical rotation inertia of the thick floors $\left(J_{f}\right)$.

Introducing the macroscopic shear force $T^{0}$ and the global bending moment $M^{0}$ already defined in Eq. (3) and accounting for $U^{0}=0$ shows that Eq. (14a) is nothing but the moment of momentum balance of the usual Timoshenko formulation:

$$
T^{0}+M^{0 \prime}=J_{f} \omega_{0}^{2} \alpha^{0}
$$

However, in massive Timoshenko beams, variables $U$ and $\alpha$ reach the dynamic regime in the same range; hence, both are involved in common modes. Conversely, for the reticulated beams studied in this section, natural and gyration modes are uncoupled because the dynamic regimes for $U$ and $\alpha$ occur in different frequency ranges. This specificity implies that in the frequency range of non-homogenizable natural modes, homogenizable gyration modes can be found. Note that the thick floors of the specific studied frame lead to neglecting the shear deformability of the floor 
and the rotation inertia of the walls. The particular description [Eq. (14)] can be extended to other types of frames by considering the cell shear stiffness $K$ instead of $K_{w}$ and rotation inertia $J$ instead of $J_{f}$ (for structures as in Fig. 2, $J=J_{f}+J_{w}$ with $\left.J_{w}=\rho_{w} A_{w} \ell_{f}^{2} / 2\right)$.

\subsection{Modal Analysis and Physical Properties}

To analyze the features of gyration modes, let us first establish the energy balance by multiplying Eq. (14a) by the rotation $\alpha$ and integrating over the beam length [superscripts 0 are omitted to save notations and, for more generality, the extended form of Eq. (14a) with $J$ and $K$ is considered]. After integration by part, one obtains:

$$
\frac{1}{2} \int_{0}^{H} J \omega^{2} \alpha^{2} d x=\frac{1}{2} \int_{0}^{H}\left(\frac{T^{2}}{K}+\frac{M^{2}}{E_{w} I}\right) d x+\frac{1}{2}[M \alpha]_{0}^{H}
$$

which shows that the working boundary conditions are moment $M$ and rotation $\alpha$, while the shear force only takes part in the energy of deformation. Hence, the external actions for which these modes are relevant are either vertical differential forces (global moment) or vertical differential displacements (section rotation) imposed at the extremities of the walls. For instance, considering a beam clamped on $x=0$ (no section rotation) and free on $x=H$ (no global moment), the boundary conditions read:

$$
\alpha(0)=0 \quad \text { and } \quad M(H)=0, \text { thus } \quad \alpha^{\prime}(H)=0
$$

Because of the presence of the source term $K \alpha$ in the moment of momentum balance [Eq. (14a)], the gyration dynamics deviates from the dynamics of systems governed by a Helmholtz equation (as shear beam). To investigate the features of gyration modes, Eq. (14a) is rewritten in the form:

$$
\alpha^{\prime \prime}=-D \alpha ; \quad D=\frac{J \omega^{2}-K}{E_{w} I} ; \quad \delta=1 / \sqrt{|D|}
$$

As $D$ may be positive or negative, the character of the gyration modes differs for frequencies lower or higher than the threshold frequency $\sqrt{K / J}$.

In the low-frequency range defined by $\omega \leq \sqrt{K / J}$, then $D<0$ and the general solution reads:

$$
\alpha(x)=A \cosh (x / \delta)+B \sinh (x / \delta)
$$

The determination of $A$ and $B$ from clamped-free boundary conditions (16) leads to the trivial solution $\alpha^{0}(x)=$ 0 . Other boundary conditions may lead to $\alpha^{0}(x) \neq 0$, with an exponential decay of characteristics length $\delta=$ $O\left(\sqrt{E_{w} I / K}\right)$ when $\omega \rightarrow 0$.

Thus, actual gyration modes only appear for frequencies higher than the threshold. Indeed:

$$
\text { for } \omega \geq \sqrt{K / J}, \quad \alpha(x)=A \cos (x / \delta)+B \sin (x / \delta)
$$

and boundary conditions (16) impose $A=0$ and $(B / \delta) \cos (H / \delta)=0$. Finally, the clamped-free gyration modes are defined by

$$
\alpha_{k}(x)=B \sin \left(x / \delta_{k}\right) ; \quad \delta_{k}=\frac{2 H}{(2 k-1) \pi} ; \quad \omega_{k}^{2}=\frac{E_{w} I}{J} \frac{(2 k-1)^{2} \pi^{2}}{4 H^{2}}+\frac{K}{J}
$$

Consequently, the macroscopic length (namely, $\tilde{L}_{k}=\delta_{k}$ ) of the $k$ th gyration mode follow the common estimate. The mode shape (in $\alpha$ ) is of the same sinusoidal nature as shear beam (in $U$ ). Conversely, the frequency distribution does not follow the series of odd numbers. 


\subsection{Numerical Illustration}

These results are illustrated and confirmed numerically on a structure (as in Fig. 2) of $N=15$ levels and of span $h=10 \mathrm{~m}$. Thus, $\tilde{\epsilon}=\pi / 2 N=0.105$. Choosing a squared frame of length $\ell_{w}=\ell_{f}=3 \mathrm{~m}$, the wall and floor thicknesses respect Eq. (9), which gives: $a_{w}=\tilde{\epsilon} \ell_{w}=31.4 \mathrm{~cm}, a_{f}=\sqrt{\tilde{\epsilon}} \ell_{w}=97.1 \mathrm{~cm}$. The material properties are those of concrete: $\rho=2500 \mathrm{~kg} / \mathrm{m}^{3}, E_{\text {Young }}=30 \mathrm{GPa}, v=0.2, E=E_{\text {Young }} /\left(1-v^{2}\right)=31.250 \mathrm{GPa}$.

As the scale separation is not very sharp, the thicknesses deduced from Eq. (9) yield that the wall inertia and shear deformability of the floor are not negligible. For this reason, the accuracy of the gyration beam model is improved by using $J$ and $K$ instead of $J_{f}$ and $K_{w}$. The frequencies of the fourth first gyration modes are deduced from Eq. (17) and compared to a finite-element (FE) modeling (RDM6). The results presented in Fig. 5 show that the discrepancies are smaller than $8 \%$. Accounting for the poor scale separation, this rather good accuracy illustrates the robustness of

mode 1

H.M. : $34.75 \mathrm{~Hz}$

F.E. : $33.09 \mathrm{~Hz}$

Error : $5.0 \%$

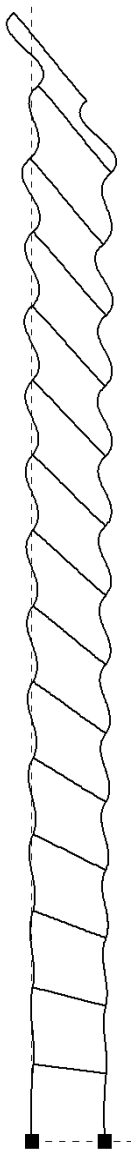

mode 2

56.96

53.61

6.3

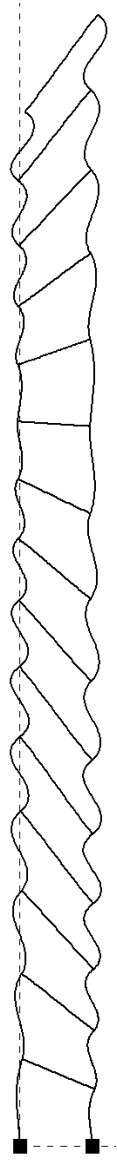

mode 3

85.54

80.13

6.8

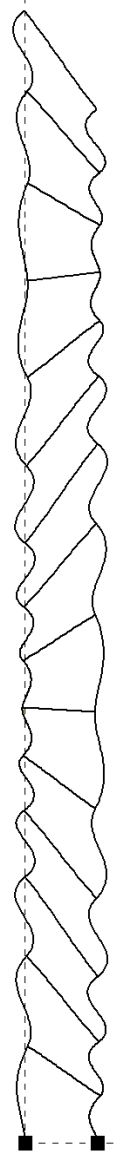

mode 4

115.88

107.66

7.6

FIG. 5: Fourth first frequencies and shapes of gyration modes calculated by RDM6. Comparison between homogenization method (H.M.) and finite elements (F.E.) and relative error. 
the homogenization approach. The mode shapes clearly evidence the specific nature of the gyration modes governed by the section rotation, with negligible transverse motions. Notice also that the gyration eigenfrequency ratio $f_{k} / f_{1}=$ $\{1 ; 1.6 ; 2.5 ; 3.3\}$ departs very significantly from the odd number series, with a much denser distribution.

\section{GENERALIZATION: COMPREHENSIVE APPROACH}

The previous section leads us to infer the existence of structures responding to more general descriptions where both translation and rotation inertias balance macro-scopic shear force $T$, global bending moment $M$, and inner bending moment $\mathcal{M}$. This generalized model is established directly at the macro-scale independently of the homogenization process. It enables recovering the results already established, but also to identifying the occurrence of gyration modes for more complex basic frames. The theoretical approach based on balance equations and beam laws is first presented, then discussed trough a dimensional analysis, and finally its validity is illustrated from a numerical example.

\subsection{Generic Beam Model Including Gyration Modes}

The model is built by introducing, as in Eq. (15), the rotation inertia in the moment of momentum balance of the generic model, while keeping the same beam behavior laws (3):

$$
\left\{\begin{array}{l}
\left(T-\mathcal{M}^{\prime}\right)^{\prime}=\Lambda \omega^{2} U ; \quad T=-K\left(U^{\prime}-\alpha\right) \\
\mathcal{M}=-E_{w} \mathcal{I} U^{\prime \prime} ; \quad M^{\prime}+T=J \omega^{2} \alpha \\
M=-E_{w} I \alpha^{\prime}
\end{array}\right.
$$

To facilitate the physical understanding of the interactions between the mechanisms involved in this model, we proceed to its dimensional analysis. In addition to the dimensionless numbers already defined in Eq. (6) we introduce the new number $\zeta$ attached to the rotation inertia:

$$
\zeta=\frac{J}{\Lambda \tilde{L}^{2}}
$$

For common geometry, $\zeta$ has a typical value of $\zeta=O\left(\ell_{f}^{2} / \tilde{L}^{2}\right)=O\left(\tilde{\epsilon}^{2}\right)$. The dimensionless formulation of Eq. (18) evidences the coupling between the dynamic operators acting on $U^{*}$ and $\alpha^{*}$ :

$$
\left\{\begin{array}{l}
\Omega^{2} U^{*}+U^{*(2)}-C \gamma U^{*(4)}=\left(\tilde{L} \alpha^{r} / U^{r}\right) \alpha^{* \prime} \\
\left(\zeta \Omega^{2}-1\right) \alpha^{*}+C \alpha^{*(2)}=-\left(U^{r} / \tilde{L} \alpha^{r}\right) U^{* \prime}
\end{array}\right.
$$

and the sixth-order differential equation driving $U^{*}$ or $\alpha^{*}$ reads:

$$
C \gamma U^{*(6)}-\left[1+\gamma\left(1-\zeta \Omega^{2}\right)\right] U^{*(4)}-\left(1+\frac{\zeta}{C}\right) \Omega^{2} U^{*(2)}+\left(1-\zeta \Omega^{2}\right) \frac{\Omega^{2}}{C} U^{*}=O(\tilde{\epsilon})
$$

In principle, it is possible to design periodic structures from cells such that all the dimensionless numbers are $O(1)$. In that case, by construction, all the mechanisms conjointly exist [and then $U^{r} /\left(\tilde{L} \alpha^{r}\right)=O(1)$; i.e., $O(\alpha)=O\left(U^{\prime}\right)$ ]. The modes of such structures involve both $U^{*}$ and $\alpha^{*}$ in the same frequency range and the classification of natural and gyration modes disappears.

By varying the properties of the basic cell, simplified forms of the model can be reached for other structures, according to the values of the dimensionless numbers in power of $\tilde{\epsilon}=\ell / \tilde{L}$. For instance, the generic beam where the rotational inertia is negligible [Eq. (7)], is obtained provided that $C$ and $\gamma$ are $O(1)$ and that:

$$
\frac{\zeta}{C}=\frac{J K}{E_{w} I \Lambda} \leq O(\tilde{\epsilon}) \quad \text { and } \quad \zeta \Omega^{2}=\frac{J \omega^{2}}{K} \leq O(\tilde{\epsilon})
$$

The first inequality concerns the mass distribution within the cell: the radius of gyration $\sqrt{J / \Lambda}$ should be smaller than the intrinsic bending/shear length $\sqrt{E_{w} I / K}$. The second condition imposes frequencies lower than the threshold 
frequency to be in the range of vanishing gyration modes (Section 4.2). Besides, one obtains a "full" Timoshenko beam (that involves at the leading order shear, global bending and rotation inertia) when:

$$
C=\frac{E_{w} I}{K \tilde{L}^{2}}=O(1) ; \quad \gamma=\frac{\mathcal{I}}{I} \leq O(\tilde{\epsilon}) ; \quad \frac{\zeta \Omega^{2}}{C}=\frac{J \omega^{2} \tilde{L}^{2}}{E_{w} I}=O(1) ; \quad \text { i.e. }, \quad \zeta \Omega^{2}=O(1)
$$

The latter requirement implies that frequencies should be higher than the threshold frequency for gyration modes. In the lower-frequency range $\zeta \Omega^{2} \leq O(\tilde{\epsilon})$ the slender Timoshenko beam analyzed in Section 3.2 is recovered. These examples show that the occurrence of gyration modes depends on both the structure of the cell (say the distribution of mass compared to the distribution of stiffness) and on the frequency range. The next section aims at defining the condition in which macroscopic gyration modes do exist.

\subsection{Condition of Existence of Gyration Modes}

A necessary condition for the existence of gyration modes is that $\alpha$ is actually in a dynamic regime. This imposes, [cf. Eq. (19b)], (1) a frequency higher than the threshold frequency of vanishing gyration, and (2) the balance between rotation inertia and moment. Consequently,

$$
\zeta \Omega^{2}=\frac{J \omega^{2}}{K} \geq O(1) ; \quad \frac{\zeta \Omega^{2}}{C}=\frac{J \omega^{2} L^{2}}{E_{w} I}=O(1) \quad \text { thus } \quad C \geq O(1)
$$

The inequality $C \geq O(1)$ provides a first criterion of existence: gyration modes can only appear in structures such that the macro-deformation is not dominated by the global bending mechanism. This condition will be assumed in the sequel.

To go further, notice that gyration modes distinct from natural modes, means that among the modes one may distinguish two uncoupled families. To identify the situations of uncoupling, it is convenient to write Eqs. (20) in the hereafter equivalent forms deduced from Eqs. (19):

$$
C\left[C \gamma U^{*(4)}-U^{*(2)}-\Omega^{2} U^{*}\right]^{(2)}+\left(\zeta \Omega^{2}-1\right)\left[C \gamma U^{*(4)}-U^{*(2)}-\Omega^{2} U^{*}\right]-U^{*(2)}=O(\tilde{\epsilon})
$$

where, according to Eq. (19a), the terms in brackets are proportional to $\alpha^{* \prime}$, or

$$
-C \gamma\left[\left(\zeta \Omega^{2}-1\right) \alpha+C \alpha^{*(2)}\right]^{(4)}+\left[\left(\zeta \Omega^{2}-1\right) \alpha+C \alpha^{*(2)}\right]^{(2)}+\Omega^{2}\left[\left(\zeta \Omega^{2}-1\right) \alpha+C \alpha^{*(2)}\right]+\alpha^{*(2)}=O(\tilde{\epsilon})
$$

where, according to Eq. (19b), the terms in brackets are proportional to $U^{* \prime}$. Two possibilities of uncoupling arise depending on the magnitude of $C$. A synthesis of the discussion developed below is presented in the $C, \gamma$ mapping (Fig. 4).

\subsubsection{Structures such that $C=O(1)$}

For structures with balanced global bending and shear mechanisms; i.e., $C=O(1)$, there are two families of modes associated to two frequency ranges, provided that $\zeta \leq O(\tilde{\epsilon})$. The requirement $\zeta \leq O(\tilde{\epsilon})$ means that the radius of gyration of the cell is smaller (of half an order at least) than the size of the whole structure, and it is generally satisfied for common framed cells.

First, consider frequencies such that

$$
\Omega^{2}=O(1) ; \quad \text { i.e., } \quad \omega^{2}=O\left(\frac{K}{\Lambda \tilde{L}^{2}}\right)
$$

then Eq. (21) reduces to

$$
\left[C \gamma U^{*(4)}-U^{*(2)}-\Omega^{2} U^{*}\right]^{(2)}-\frac{1}{C}\left[C \gamma U^{*(4)}-\Omega^{2} U^{*}\right]=O(\tilde{\epsilon})
$$


This latter equation coincides with Eq. (7) that governs the natural modes of the generic beam model. Notice that the kinematics of these modes also involves a rotation $O(\alpha)=O\left(U^{\prime}\right)$.

Now, at higher frequencies such that

$$
\zeta \Omega^{2}=O(1) ; \quad \text { i.e., } \quad \omega^{2}=O\left(\frac{K}{J}\right)
$$

Then $\Omega^{2} \geq O\left(\tilde{\epsilon}^{-1}\right)$ and Eq. (22) reduces to the equation that governs the gyration modes:

$$
\frac{1}{C}\left(\zeta \Omega^{2}-1\right) \alpha+\alpha^{*(2)}=O(\tilde{\epsilon})
$$

From Eq. (19a) the translation satisfies $O(U)=O\left(\tilde{L} \alpha^{r} \alpha^{* \prime} / \Omega^{2}\right)=O\left(\alpha / \Omega^{2}\right) \ll O(\alpha)$. Consequently, in this frequency range, occur pure gyration modes.

This situation corresponds to the example treated in Section 4.2 where in addition a weak inner bending mechanism was assumed; i.e., $\gamma \leq O(\tilde{\epsilon})$. This latter assumption does not interfere with the above description of gyration modes, however, at low frequency, occur the natural modes of a slender Timoshenko beam driven by

$$
U^{*(4)}+\Omega^{2} U^{*(2)}-\frac{\Omega^{2}}{C} U^{*}=O(\tilde{\epsilon})
$$

\subsubsection{Structures such that $C \geq O\left(\epsilon^{-1}\right)$}

Consider now structures with negligible global bending mechanism compared to shear, so that $C \geq O\left(\epsilon^{-1}\right)$. As a consequence Eq. (21) simplifies to

$$
\left[C \gamma U^{*(4)}-U^{*(2)}-\Omega^{2} U^{*}\right]^{(2)}+\frac{\zeta \Omega^{2}}{C}\left[C \gamma U^{*(4)}-U^{*(2)}-\Omega^{2} U^{*}\right]=O(\tilde{\epsilon})
$$

Without evoking the frequency ranges, Eq. (23) splits into two uncoupled equations. Indeed, either the terms in brackets vanish, which leads to the fourth degree differential operator driving the natural modes of a shear-inner bending beam, or the terms in brackets, proportional to $\alpha^{* \prime}$, satisfy the second degree differential operator driving the gyration modes:

$$
\left\{\begin{array}{l}
\Omega^{2} U^{*}+U^{*(2)}-C \gamma U^{*(4)}=O(\tilde{\epsilon}) \\
\frac{\zeta \Omega^{2}}{C} \alpha^{*}+\alpha^{*(2)}=O(\tilde{\epsilon})
\end{array}\right.
$$

The natural modes appear for frequency $\omega^{2}=O\left[E_{w} \mathcal{I} /\left(\Lambda \tilde{L}^{4}\right)\right]$ if $C \gamma \gg 1$, or $\omega^{2}=O\left[K /\left(\Lambda \tilde{L}^{2}\right)\right]$ if $C \gamma \leq 1$.

The gyration modes appear at frequencies $\omega^{2}=O\left[E_{w} I /\left(J \tilde{L}^{2}\right)\right]$. The condition $\zeta<1$ is sufficient for the frequency range of gyration modes to be higher than those of natural modes. Note that, conversely to the previous case, the source term $(K \alpha$ in dimensional form) vanishes in the moment of momentum balance [Eq. (24b)]. Consequently, here, the gyration modes follow the classical features of systems driven by an Helmholtz equation, namely sinusoidal mode shapes (in $\alpha$ ), frequency distribution according to the odd number series and no threshold frequency. This second situation is illustrated in the next section.

\subsection{A Numerical Validation}

The validity of the above developments has been checked numerically on a (fictitious) structure of $N=15$ levels ( $\tilde{\epsilon}=\pi / 2 N=0.105$ ) and of span $h=10 \mathrm{~m}$. The cell is built in order to satisfy both Eqs. (24) in the same frequency range. To enhance the rotation inertia, the cell is made of a double frame where the lengths of walls and floors are, respectively, $\ell_{w}=3 \mathrm{~m}$ and $\ell_{f}=6 \mathrm{~m}$. To favor the inner bending, the thickness of both external and internal walls is 
$a_{w}=80 \mathrm{~cm}$, and that of floors is $a_{f}=20 \mathrm{~cm}$. In addition, the density of each external wall is increased to $\rho=10$ $000 \mathrm{~kg} / \mathrm{m}^{3}$, while that of the floors and internal wall is decreased to $\rho=500 \mathrm{~kg} / \mathrm{m}^{3}$. The modulus is that of concrete (see Section 4.3). With these values the scaling of the dimensionless numbers is

$$
C=O\left(\tilde{\epsilon}^{-2}\right) ; \quad C \gamma=O(\tilde{\epsilon}) ; \quad \zeta=O(\tilde{\epsilon})
$$

Figure 1 displays the transverse modes calculated through RDM6. The kinematics shows the uncoupling of both category of modes. As continuous modeling is only valid up to $N / 3=5$ modes, the eigenfrequencies of first gyration modes appear beyond the homogenizable domain of the natural modes. This is even more clear for the second gyration mode. Besides, as expected from Eq. (24b), [or also Eq. (17) when $C \gg 1$ ], the second gyration frequency is very close to three times the first gyration frequency. Again, even with a poor scale separation, the prediction of the homogenized model seems rather reliable. Finally, it is worth underlining the drastic differences between the mechanical characteristics of the cells constituting this structure and the structure studied in Section 4.3, while both clearly exhibit gyration modes.

\section{CONCLUSION}

Reticulated beams present much more diversified behaviors than massive homogeneous beams. This results from the large contrast of the shear and extension deformabilities of the cell. The homogenization method applied to single framed structures, whose results are extended to a wider class of reticulated structures through dimensional analysis, provides an efficient tool to identify the different families of behaviors.

In particular, this paper focuses on the possibility of gyration modes only based on the moment of momentum balance of the section, conversely to the natural modes. Such atypical mode kinematics, derived theoretically and proven numerically for reticulated beams, are impossible in usual beams. Indeed, for this latter, the scale ratio matches the slender ratio $\tilde{\epsilon}=b / H$ where $b$ is the section size, and $\gamma=0, \zeta=O\left(\tilde{\epsilon}^{2}\right), C=O\left(\tilde{\epsilon}^{2}\right)$, and $\zeta / C=O(1)$. Consequently, the gyration modes would appear at frequencies where the shear wavelength of the beam material is of the order of section size $b$, which is in contradiction with a beam modeling. For the same reason, the Timoshenko formulation in usual beams is necessarily a corrector (associated to weak terms) of the classical Euler-Bernoulli beam. Conversely, for reticulated beams made of specific cells, all the terms can be of the same order, and their features are far from that of Euler-Bernoulli beams.

The study underlines that gyration modes generally occur at higher frequencies that natural modes. For this reason mechanisms of inner resonance (by bending of the elements) may conjointly occur, Chesnais et al. (2007), Chesnais (2010). For instance, this would be the case for framed structures whose cell geometry is scaled by $O\left(a_{w} / \ell_{w}\right)=$ $O\left(a_{f} / \ell_{w}\right)=O(\epsilon)$. This situation, not considered in the present paper, leads to the same global moment parameters than for local quasi-statics but differs fundamentally by the inertia term and the inner elastic source of moment, both depending on frequency. Again, this behavior is impossible in massive beams.

Numerical modeling performed on two structures with very different inner morphologies of the cell (hence, mechanical characteristics) confirms the theoretical results. Besides, dynamic experiments on real regular buildings (mostly based on ambient noise records) Boutin et al. (2005) have demonstrated the reliability of the homogenization method coupled with a systematic use of scaling and its practical interest in structural dynamics. However, in this domain, according to loading principally due to the base translation, the observations focus on the analysis of natural modes and disregard motions generated by much noisier external loading.

More generally, to our knowledge, no experimental evidence of gyration modes is available in the literature. While they are undoubtedly uncommon, they could be of importance for structures anchored in rotating bases or for structures loaded by vibrating equipment or machinery generating (parasite) momentum. A direct application of the present study is to provide simple criteria enabling to identify the reticulated structures in which gyration modes may occur and to specify the external actions for which they are relevant. Thus, in complement to the numerical validation, simple reticulated systems may be designed to confirm experimentally the unusual features of these modes. The investigation of the practical applications of a dense frequency distribution of macro-modes, in a frequency range where the natural modes have lost their spatial coherence by absence of scale separation, would also be of interest. 
Finally, these results may be extended to the rheology of reticulated materials such as foam, glass wool, plant, bones, etc. Further, they present strong analogies with generalized continua (Eringen, 1968; Boutin et al., 2009).

\section{ACKNOWLEDGMENTS}

The authors thank Professor Yves Debard for giving free access to the code RDM6.

\section{REFERENCES}

Boutin, C. and Hans, S., Homogenisation of periodic discrete medium: Application to Dynamics of framed structures, Comput. Geotech., vol. 30, no. 4, pp. 303-320, 2003.

Boutin, C., Hans, S., Ibraim, E., and Roussillon, P., In situ experiments and seismic analysis of existing buildings. Part II, Earthquake Eng. Struct. Dyn., vol. 34, pp. 1531-1546, 2005.

Boutin, C., Hans, S., and Chesnais, C., Generalized beams and continua. Dynamics of reticulated structures, Proc. of Euromech Colloquium 510 Mechanics of Generalized Continua: A Hundred Years after the Cosserats, May 13-16 Paris, eds. Maugin, G. and Metrikine, A. V., Berlin: Springer, 2009.

Caillerie, D., Trompette, P., and Verna, P., Homogenisation of periodic trusses, Proc. of Congress IASS, Madrid, pp. 7139-7180, 1989.

Chesnais, C., Dynamique de milieux réticulés non contreventés, Application aux bâtiments, PhD thesis, École Nationale des Travaux Publics de l'État (ENTPE), Lyon, 2010.

Chesnais, C., Hans, S., and Boutin, C., Wave propagation and diffraction in discrete structures: Effect of anisotropy and internal resonance, Proc. of International Congress on Industrial and Aplied Mathematics (ICIAM), July 16-20, Zurich, 2007.

Cioranescu, D. and Saint Jean Paulin, J., Homogenization of Reticulated Structures, Applied Mathematical Sciences, vol. 136, New York: Springer, 1999.

Eringen, A. C., Mechanics of micromorphic continua, Proc. of International Union of Theoretical and Applied Mechanics (IUTAM) Symposium on the Generalized Cosserat Continuum and the Continuum Theory of Dislocations with Applications, Berlin: Springer, pp. 18-35, 1968.

Hans, S. and Boutin, C., Dynamics of discrete framed structures: A unified homogenized description, J. Mech. Mater. Struct., vol. 3, no. 9, pp. 1709-1739, 2008.

Kerr, A. D. and Accorsi, M. L., Generalization of the equations for frame-type structures-a variational approach, Acta Mech., vol. 56, no. 1-2, pp. 55-73, 1985.

Moreau, G. and Caillerie, D., Continuum modeling of lattice structures in large displacement applications to buckling analysis, Comput. Struct., vol. 68, no. 1-3, pp. 181-189, 1998.

Noor, A. K., Continuum modeling for repetitive lattice structures, Appl. Mech. Rev., vol. 41, no. 7, pp. 285-296, 1988.

Sanchez-Palencia, E., Non-Homogeneous Media and Vibration Theory, Lecture Note in Physics, vol. 127, Berlin: Springer, 1980.

Skattum, K. S., Dynamic analysis of coupled shear walls and sandwich beams, Report No. EERL71-06, California Institute of Technology, Pasadena, CA, 1971.

Tollenaere, H. and Caillerie, D., Continuous modeling of lattice structures by homogenization, Adv. Eng. Software, vol. 29, no. 7-9, pp. 699-705, 1998. 